\title{
LYMPH NODE MICROMETASTASES OF ADENOCARCINOMA LOCATED IN THE GASTROESOPHAGEAL JUNCTION
}

\author{
Janusz WeodarczyK ${ }^{1}$, James MuelleR ${ }^{2}$, JoAnna WeodarczyK ${ }^{3}$
}

1Department of General Thoracic Surgery, John Paul II Hospital, Cracow, Poland

2Department of Pathology, Baystate Medical Center, Springfield, Massachusetts USA

${ }^{3}$ The $2^{\text {nd }}$ Department of Internal Medicine, J. Dietl Memorial Specialist Hospital, Cracow, Poland

\begin{abstract}
Background: The aim of this study was to evaluate the presence of micrometastases (MM) and tumor cell microinvolvement (TCM) in regional lymph nodes of patients with gastroesophageal junction adenocarcinoma.

Material and methods: 61 patients with adenocarcinoma (stage N0) were included in this study. Lymph nodes removed during surgery were examined using mixed monoclonal antibodies against pan-cytokeratins (AE1/AE3). Micrometastases was defined as single neoplastic cells or infiltration with diameter below $0.5 \mathrm{~mm}$ but not involving the interstitium.

Results: Micrometastases were found in 2 cases with adenocarcinoma type I and 4 cases with type III. Tumor cell microinvolvement was found in 2 cases of either adenocarcinoma type I or type III (according to Siewert's classification). Presence of MM did not influence survival rate.

Conclusions: Despite no influence of MM presence on survival rate, MM came from adenocarcinoma type III more frequently. The presence of MM proved lack of homogeneity in the group of N0 stage and makes evaluation advantageous.
\end{abstract}

Key words: micrometastasis, adenocarcinoma gastroesophageal junction.

\section{Introduction}

Morbidity of adenocarcinoma located in the gastroesophageal junction has increased within the last decade $[1,2]$. Radical surgery of tumor with removal of lymph nodes is now routine therapy. Regardless of accepted treatment, the survival rate is still not satisfactory [3]. Metastases to lymph nodes are claimed to be independent and one of the most important prognostic factors in gastroesophageal junction adenocarcinoma $[4,5]$. Local recurrences of disease or even disease spread are observed in patients without metastases in routinely examined lymph nodes as well. Thanks to modern immunohistochemical techniques using monoclonal antibodies which expose micrometastasis $(\mathrm{MM})$, diagnosis and treatment of cancer can be improved. These new methods have already been tested in diagnosis of lung cancer, colon cancer, gastric and esophageal cancer [6-8]. Some authors have reported use of monoclonal antibodies for MM assessment as an independent prognostic factor for survival rate in neoplastic disease [9, 10].

\section{Material and methods}

\section{Patients}

Sixty-one patients with confirmed adenocarcinoma of the gastroesophageal junction were included in the study. According to the Siewert and Stein classification [11] they were divided into three groups: patients with (AEG type I - adenocarcinoma related to Barrett esophagus (AEG type I) (32 cases), adenocarcinoma of cardia (AEG type II), and subcardial adenocarcinoma (AEG type III) (29 cases). Only patients with type I and type III adenocarcinoma were included in this study. The TNM UICC classification was used for assessment of the disease stage [12]. Patients with AEG1 tumors underwent transmediastinal esophagectomy, proximal stomach and lymphadenectomy in the posterior me- 
diastinum and celiac axis. An extended total gastrectomy with resection of the distal esophagus and D3 lymphadenectomy with transhiatal lymphadenectomy of the inferior mediastinum was the procedure for patients with AEG3 tumors. In total 1130 lymph nodes were removed during surgery, on average 22 lymph nodes per patient (range 8-58 lymph nodes). In 60 cases surgery was radical (R0) while 1 case which was classified as $\mathrm{R} 1$. The mean follow-up time for the 61 patients was 27.4 months, with a range 1-96.7 months. Table I shows clinical and histopathologic outcomes of patients.

\section{Immunohistochemistry}

The expression of epithelial antigens was determined in formalin-fixed paraffin embedded slides. The sections, $4 \mu \mathrm{m}$ thick, were deparaffinized in xylene and hydrated in alcohol. Endogenous peroxidase was blocked with $3 \%$ peroxidase for 10 minutes. Protein blocking was done with a protein blocker to reduce non-specific binding of primary and secondary antibodies. Next, the slides were incubated with anti-cytokeratins cocktail (AE1/ AE3, Boehringer, Mannheim, Germany) and anti-BerEP4 antibody (DAKO Diagnostica, Hamburg, Germany), at 1 : 500 dilution. The use of a detection system was followed by visualization of the antigenantibody complex using chromogen 3,3 diaminoben-

Table I. Clinical and pathomorphological data of patients with AEG1 and AEG3

\begin{tabular}{|c|c|c|c|}
\hline GROUP & AEG1 & AEG3 & TOTAL \\
\hline $\mathrm{N}$ & 32 & 29 & 61 \\
\hline Sex $M: F$ & $26: 6$ & $23: 6$ & 61 \\
\hline Age & $45-75$ & $33-78$ & $33-78$ \\
\hline Follow-up & $27.7(1-96.7)$ & $26.6(1-91.7)$ & $27.4(1-96.7)$ \\
\hline $\begin{array}{l}\text { Differentiation } \\
\text { grade } \\
\text { G1: G2 : G3 }\end{array}$ & $\begin{array}{l}n 1: 16: 15 \\
3\end{array}$ & $0: 16: 13$ & $1: 32: 28$ \\
\hline $\begin{array}{l}\text { Lauren type } \\
(\mathrm{I}: \mathrm{M}: \mathrm{D})\end{array}$ & $26: 3: 2$ & $19: 4: 7$ & $45: 7: 9$ \\
\hline \multirow[t]{10}{*}{ TNM } & T1N0M0 11 & T1N0M0 5 & T1N0M0 16 \\
\hline & T2N0M0 3 & T2N0M0 6 & T2N0M0 9 \\
\hline & T3N0M0 2 & T3N0M0 1 & T3N0M0 3 \\
\hline & T1N1M0 1 & T1N1M0 1 & T1N1M0 2 \\
\hline & T2N1M0 6 & T2N1M0 8 & T2N1M0 14 \\
\hline & T3N1M0 7 & T3N1M0 1 & T3N1M0 8 \\
\hline & T2N2M0 2 & T4N1M0 1 & T4N1M0 1 \\
\hline & \multirow[t]{3}{*}{32} & T2N2M0 1 & T2N2 M0 3 \\
\hline & & T3N2M0 5 & T3N2 M0 5 \\
\hline & & \multicolumn{2}{|c|}{$\begin{array}{ll}29 & 61 \\
\end{array}$} \\
\hline
\end{tabular}

AEG 1 - adenocarcinoma gastroesophageal junction type 1

AEG 3 - adenocarcinoma gastroesophageal junction type 3

Lauren (I:M:D) - Lauren (intestinal : mixed: diffuse) zidine (DAB). Histopathologic criteria used for diagnosis of micrometastasis in lymph nodes:

Micrometastasis (MM) is defined as tumor cells or cell cluster less than $0.5 \mathrm{~mm}$ of maximal diameter within the lymph node.

Tumor cell involvement (TCM) is defined as freefloating neoplastic cells or cell clusters within the subcapsular sinus or intramedullary sinuses of the lymph node.

Metastases are defined as neoplastic focus exceeding $0.5 \mathrm{~mm}$ in diameter.

\section{Data analysis}

Relationships between groups were calculated by Cox test. For assessment of morbidity Kaplan-Meier test was used. A P $<0.05$ was considered statistically significant.

\section{Results}

Lymph nodes obtained from 28 patients with N0 stage of disease were examined for the presence of micrometastases. Micrometastasis were found in $6(21.4 \%)$ patients and histopathologic examination showed that $4(14.3 \%)$ patients had adenocarcinoma type III and 2 patients (7.1\%) had type I. Tumor cell microinvolvement was found in $4(14.3 \%)$ cases with type I and in 2 cases $(7.1 \%)$ with type III adenocarcinoma (Table II). Occurrence of MM did not correlate either with $\mathrm{T}$ grade or tumor grade or with neoplasm type according to Lauren' classification. Micrometastases were found more frequently in type III than type I adenocarcinoma, but this difference was not statistically significant. There was no difference in survival time between patients with MM and patients with N0 grade, MM and M1 as well as TCM and pN0. Patients with metastases which exceed $0.5 \mathrm{~mm}$ in diameter were included in group $\mathrm{N} 1$.

\section{Discussion}

The assessment of micrometastases in routine $\mathrm{HE}$ staining demonstrates false negative rates. Immuno-

Table II. Distribution of patients according to $\mathrm{T}$ grade

\begin{tabular}{lcccccc}
\hline CATEGORY & \multicolumn{3}{c}{ AEG1 } & \multicolumn{3}{c}{ AEG3 } \\
\hline $\begin{array}{l}\text { PN0 cases with } \\
\text { MM TCM }\end{array}$ & \multicolumn{3}{c}{ MM TCM } & MM TCM \\
\hline $\mathrm{T} 1$ & 11 & 1 & 1 & 5 & 1 & 1 \\
\hline $\mathrm{T} 2$ & 3 & 1 & 1 & 6 & 2 & 1 \\
\hline $\mathrm{T} 3$ & 2 & & & 1 & 1 & \\
\hline total & 16 & 2 & 2 & 12 & 4 & 2 \\
\hline $\begin{array}{l}\text { MM - micrometastasis, TCM - tumor cell microinvolvement, AEG 1-adeno- } \\
\text { carcinoma gastroesophageal junction type 1, AEG 3-adenocarcinoma } \\
\text { gastroesophageal junction type 3 }\end{array}$ &
\end{tabular}




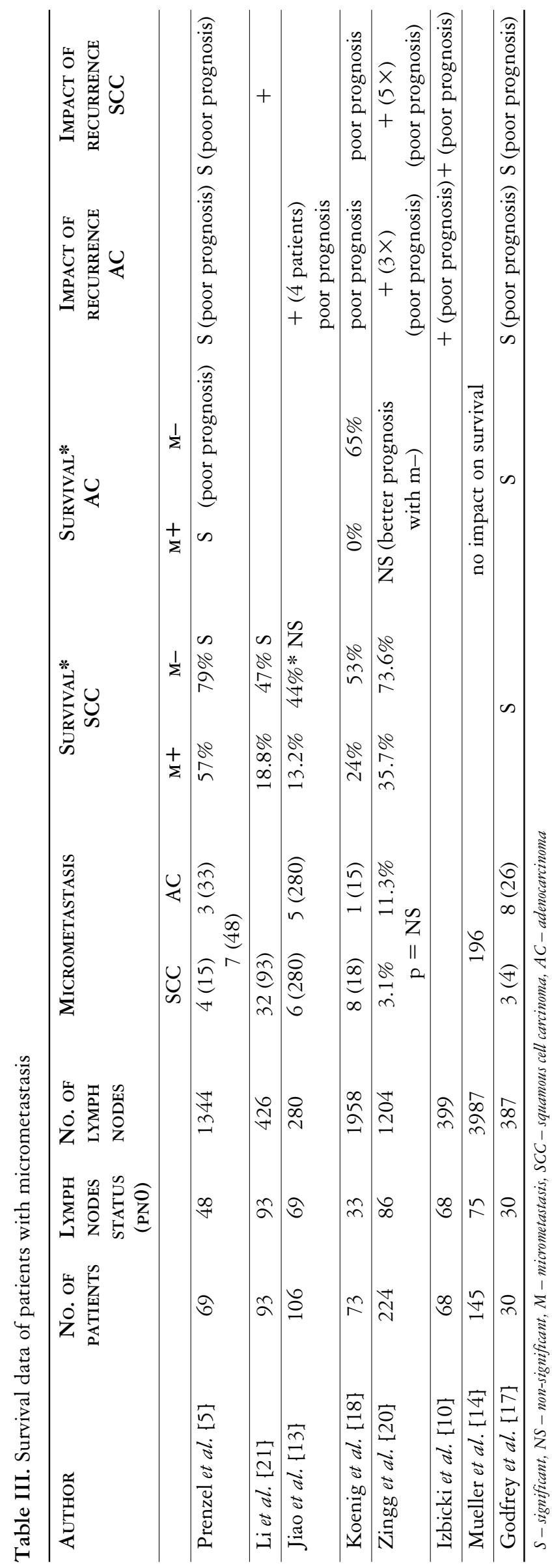

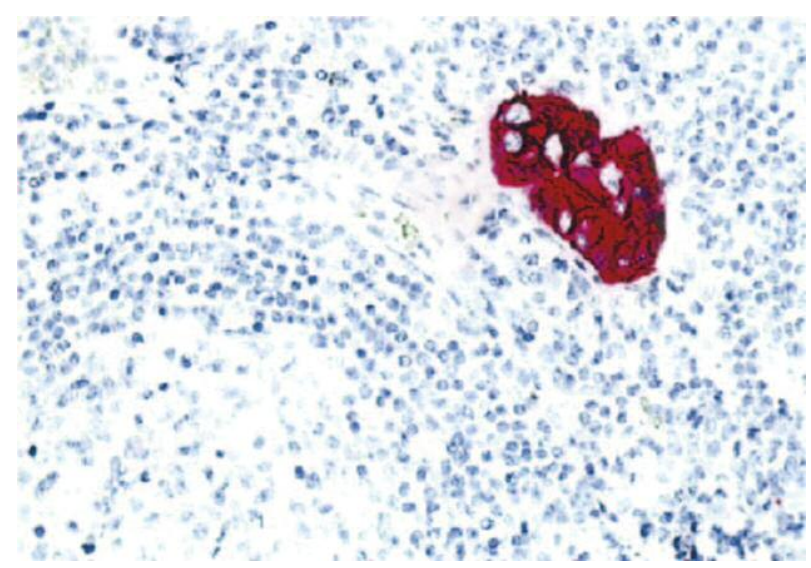

Fig. 1. Micrometastasis (MM) in a lymph node from a patient with adenocarcinoma of the esophagus. This section was stained with the antibody cocktail AE1/AE3. A cluster of positively stained tumor cells in medulla of the lymph node

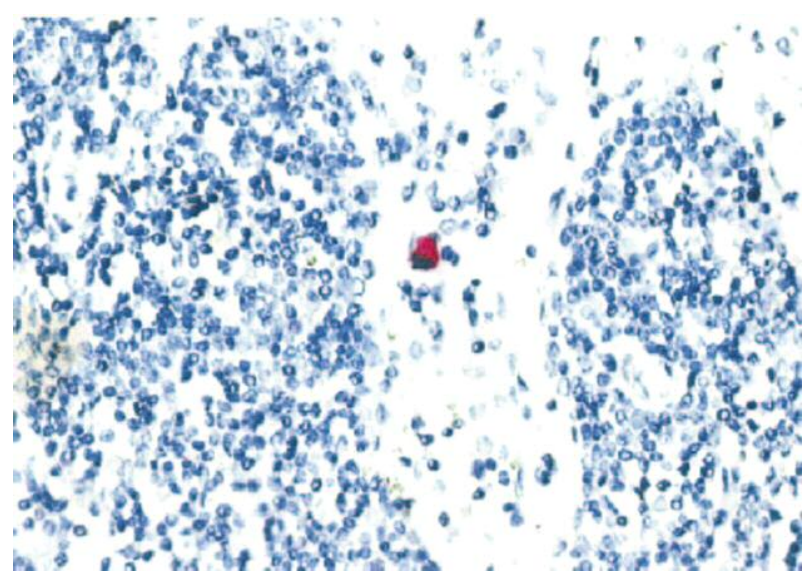

Fig. 2. Tumor cell microinvolvement (TCM) in a lymph node of adenocarcinoma of the esophagus. This section was stained with the antibody cocktail AE1/AE3.

A tumor cell without a surrounding sinus of the lymph node

histochemistry corrects detection outcome of micrometastases in esophagus cancer and identifies them in patients with negative lymph nodes in routine $\mathrm{HE}$ (11.9-30\%); also molecular examination (RT-PCR) shows a high degree of sensitivity and specificity, $100 \%$ and $67 \%$ respectively $[13-18]$.

The impact of micrometastases on the survival and progression of esophageal cancer both in adenocarcinoma (ACC) and squamous cell carcinoma (SCC) remains unclear. A lot of authors conclude micrometastases to be a poor prognostic implication both for survival and relapse (Table III). Mouriguchi suggests that this is a risk factor of neoplastic disease recurrence spread by blood [19]. Prenzel et al. reported that in patients in the early stage of carcinoma of the esophagus (SCC and ACC - pT1N0), micrometastasis applied to $15 \%$ and only those with submucosal infiltration, but were not present in patients with mucosa infiltration. The authors suggest that the lamina muscularis mu- 
cosa represents a lymphatic barrier to the submucosa. The survival of pN0 was significantly worse compared to patients with tumor free lymph nodes, and also the 5-year survival rate of the $\mathrm{pN}+$ group was still inferior compared to the micrometastasis group [5]. Analyzing the patients with SCC and ACC, Koenig et al. claim that the survival of patients in group: N0, low to $11 \%$, and high than $11 \%$ of micrometastasis is shorter than the patients in group N0, 43, 27,11 months respectively [18]. Zingg et al. found that patients with micrometastases in SCC live shorter, which is not observed in patients with ACC. They name four factors that significantly affect disease-free survival: pretreatment (radio-chemotherapy), micrometastasis, UICC stage II, and adenocarcinoma [20]. Li et al. present similar observations. The author observed the appearance of micrometastases in $9.4 \%$ of examined lymph nodes but together with the feature $T$ they constitute an independent prognostic factor in the overall 5-year survival rate of the patients [21].

Some authors point out that hidden micrometastasis should be defined as every metastasis found in classic microscopic study $[14,22]$. In our study MM were present in $21.4 \%$ of cases and were found in adenocarcinoma type III more often than in type I tumors. Additionally, MM did not influence survival time. Glickmann et al. reported MM presence in $15(31 \%)$ cases of adenocarcinoma and in $5(17 \%)$ cases of squamous cell carcinoma (the total number of cases was 78 , including 49 cases of adenocarcinomas and 29 cases of squamous cell carcinoma). He found no influence of MM on survival time, which is why he did not recommend the assessment of MM as a method for evaluation of prognosis in oesophageal cancer [15]. Stachura et al. during microscopic assessment of lymph nodes obtained from 40 patients with early stage of gastric cancer reported MM in 3 cases and metastases to lymph nodes in 3 cases as well. Presence of MM was not related to the grade of neoplasm. The authors did not observe an influence of MM on survival time as well but they suggested that MM may be a prognostic factor according to the new concept for distal metastases development [23]. Bonavina et al. studied 46 patients with adenocarcinoma of the gastroesophageal junction. They reported micrometastases in $6(33.3 \%)$ cases from a group of 18 patients with N0 stage of disease. The authors claim that despite the fact of performed radical treatment neoplasm recurrence happened in 3 cases with MM within 12 months [9]. Lee et al. confirmed a detrimental influence of MM on survival rate in patients operated on due to gastric carcinoma [24]. Presence of $\mathrm{MM}$ in lymph node indicated by the immunohistochemical method and missed in routine microscopic assessment of HE slides indicates heterogeneity of N0 variable. Patients with MM were reclassified and included in group NO [15]. In this meaning MM becomes a differentiating factor in neoplasm progression and determines therapeutic decisions. Mueller et al. did not prove an influence of MM on survival time in adenocarcinoma cases depending on carcinoma type (I, II, or III). They reported increased occurrence of MM in type II and III carcinoma in relation to type I. Some authors suggest use of a different strategy of lymphadenectomy in relation to adenocarcinoma type [14]. Meachara et al. think that patients with cytokeratin positive reaction in lymph nodes require monitoring and an individual therapeutic process [25]. In a previous paper, Izbicki et al. reported occurrence of $50 \% \mathrm{MM}$ in 68 studied carcinoma cases and significant changes of survival time. However, lack of homogeneity in the studied group, difficulties in assessment of patients with adenocarcinoma or squamous cell carcinoma markedly affected final results [10]. The authors suggest that these results may indicate patients who require adjuvant therapy, especially patients with remnant neoplasm who can respond to antineoplastic therapy more effectively than patients with a higher stage of disease. The occurrence of micrometastases is relative to early stage of neoplasm progression. Higashi analyzed 95 cases with submucosal gastric carcinoma and stated that $23(24 \%)$ patients at stage N0 had micrometastases [25]. Tanabe et al. found micrometastases in 34 patients from a group of 78 cases with superficial squamous cell carcinoma of the esophagus and occurrence of neoplastic infiltration of mucosal veins correlated with it [26].

Immunohistochemistry and molecular examination are efficient methods of micrometastasis diagnosis but technically detailed evaluation of all removed lymph nodes is not always possible. That is why the efforts of mapping SN are made. Mapping SN and its immunohistochemical and/or molecular evaluation can not only have an impact on the detection outcome correction of micrometastases but also on the limitation of lymphadenectomy in patients in the early stage of esophageal cancer [27-29].

Immunohistochemistry and molecular examination are efficient diagnostic tools in the evaluation of the staging of esophageal cancer. Precise examination not only lets us estimate the real stage of neoplasm but also helps in applying the proper therapeutic strategy. Occurrence of micrometastases in lymph nodes is a differentiating feature for stage N0. These alterations were found more often in type III adenocarcinoma than type I and they may suggest biological difference of these neoplasms or give evidence of neoplasm recurrence. Use of cytokeratin for lymph node assessment helps to estimate precisely the grade of progression of neoplastic disease and may be a key in making the therapeutic decision and follow-up for patients. Taking into consideration both the increase of the incidence of carcinoma of the esophago-gastric junction and its still poor prognosis, the precise diagnostics enables the improvement of staging of esophageal cancer. Negative lymph nodes in rou- 
tine HE should not assure us of the absence of illness but persuade us to extend the diagnostics with immunohistochemistry. The examination should be the diagnostic canon in the post-treatment evaluation of patients with esophageal cancer.

\section{The authors declare no conflict of interest.}

\section{References}

1. Altorki NK, Olveria S, Schrump DS. Epidemyology and molecular biology of Barrett's adenocarcinoma. Semin Surg Oncol 1997; 13: 270-280.

2. Sehdev A, Catencci DV. Gastroesophagalcancer: focus on epidemiology, classification, and staging. Discov Med 2013; 16: 103-111.

3. Siewert JR, Stein H. Lymphadenectomy for esophageal cancer. Langenbecks Arch Surg 1999; 384: 141-148.

4. Isozaki H, Okajima K, Fuji K. Histological evaluation of lymph nodes metastasis on serial sectioning in gastric cancer with radical lymphadenectomy. Hepato-Gastroenetrology 1997; 44: 1133-1136.

5. Prenzel KL, Hólscher AH, Drebber U, et al. Prognostic mpact factor of nodal micrometastasis in early esophageal cancer. Eur J Surg Oncol 2012; 38: 314-318.

6. Passlick BB, Izbicki JR, Kobuschok B, et al. Immunohistochemical assesment of individual tumor cells in lymph nodes with non-small-cell lung cancer. J Clin Oncol 1994; 12: 1827-1832.

7. Liefers GJ, Cleton-Jansen AM, van de Velde CJH, et al. Micrometastasis and survival in stage II coloreectal cancer. $\mathrm{N}$ Engl J Med 1998; 339: 223-228.

8. Ishida $\mathrm{K}$, Katsuyama $\mathrm{T}$, Sugiyama $\mathrm{A}$, et al. Immunohistochemical evaluation of lymph nodes micrometastases from gastric carcinomas. Cancer 1997; 79: 1069-1076.

9. Bonavina L, Ferrero S, Midolo V, et al. Lymph node micrometastasis in patients with adenocarcinoma of the esophagogastric junction. J Gastrointest Surg 1999; 3: 468-476.

10. Izbicki JR, Hosch SB, Pichlmeier U, et al. Prognostic value of immunohistochemically identifable tumor cells in lymph nodes of patients with completely resected esophageal cancer. N Engl J Med 1997; 337: 1188-1194.

11. Siewert RJ, Stein HJ. Classification of carcinoma of the esophagogastric junction. Br J Surg 1998; 85: 1457-1459.

12. UICC-International Union Against Cancer. TNM classification of malignant tumours. J Wiley and Sons, New York 2010.

13. Jiao X, Eslami A, Ioffe $\mathrm{O}$, et al. Immunohistochemistry analysis of micrometastasis in pretreatment lymph nodes from patients with esophageal cancer. Ann Thorac Surg 2003; 76: 996-1009

14. Mueller JD, Stein HJ, Qyang T, et al. Frequency and clinical impact of lymph node micrometastasis and tumor cell microinvolvement in patients with adenocarcinoma of the esophagogastric junction. Cancer 2000; 89: 1874-1882.

15. Glickamnn JN, Tores C, Wang, et al. The prognostic significance of lymph node micrometastasis in patients with esophageal carcinoma. Cancer 1999; 85: 769-778.

16. Makino H, Tajiri T, Onda M, et al. Detection of limph node micrometastasis in esophageal carcinoma. Hepatogastroenetrology 2003; 50: 1407-1410.

17. Godfrey TE, Raja S, Finkelstein SD, et al. Prognostic value of quantitative reverse transcription-polymerase chain reaction in lymph node-negative esophageal cancer patients. Clin Cancer Res 2001; 7: 4041-4048.

18. Koenig AM, Prenzel, KL, Bogoevski D, et al. Strong impact of micrometastatic tumor cell load in patients with esophageal carcinoma. Ann Surg Oncol 2009; 16: 454-462.
19. Moriguchi S, Maechara Y, Kornaga D, et al. Risk factors which predict pattern of reccurence after curative surgery for patients with advanced gastric cancer. Surg Oncol 1992; 1: 341-346.

20. Zingg U, Montani M, Busch M, et al. Prognostic influence of immunohistochemically detected lymph node micrometastasis and histological subtype in $\mathrm{pN} 0$ oesophaggeal cancer. Eur J Surg 2009; 35: 593-599.

21. Li SH, Wang Z, Liu XY, et al. Lymph node micrometastasis: a predictor of early tumor relps after complete resection of histologically node - negative esophageal cancer. Surg Today 2007; 37: 1047-1052.

22. International (Ludwig) Breast Cancer Study Group: Prognostic importance of occult axillary lymph node micrometastasis from breast cancers. Lancet 1990; 335: 434-435.

23. Stachura J, Zembala M, Heitzman J, et al. Lymph node micrometastasis in early gastric carcinoma alone inadequately reflect the new model of metastatic development. Pol J Pathol 1998; 49: 155-157.

24. Lee E, Chae Y, Kom I, et al. Prognostic relevance of immunochistochemically detected lymph node micrometastais in patients with gastric carcinoma. Cancer 2002; 94: 2867-2873.

25. Higashi H, Natsugoe S, Ishigami S, et al. Distribution of lymph node metastasis including micrometastasis in gastric cancer with submucosal invasion. World J Surg 2003; 27: 455-459.

26. Tanabe T, Nishimaki T, Watanabe H, et al. Immunochistochemically detected micrometastasis in lymph nodes from superficial esophageal squamos cell carcinoma. J Surg Oncol 2003; 82: 153-159.

27. Takeuchi H, Kawakubo H, Takeda F, et al. Sentinel node navigation in early-stage esophageal cancer Ann Thorac Cardiovasc Surg 2012; 18: 306-313.

28. Takeuchi H, Kitajima M, Kitagawa Y. Sentinel lymph node as a target of molecular diagnosis of lymphatic micrometastasis and local immunoresponse to malignant cells. Cancer SCI 2008; 99: $441-450$

29. Hagihara T, Uenosono Y, Arigami T, et al. Assessment of setninel node concept in esophageal cancer based on lymph node micrometastasis Ann Surg Oncol 2013; 20: 3031-3037.

\section{Address for correspondence}

\section{Janusz Włodarczyk}

Department of General Thoracic Surgery

John Paul II Hospital

Prądnicka 80

31-202 Krakow

tel. +48126142028

fax +48126143431

e-mail: jr.wlodarczyk@gmail.com 